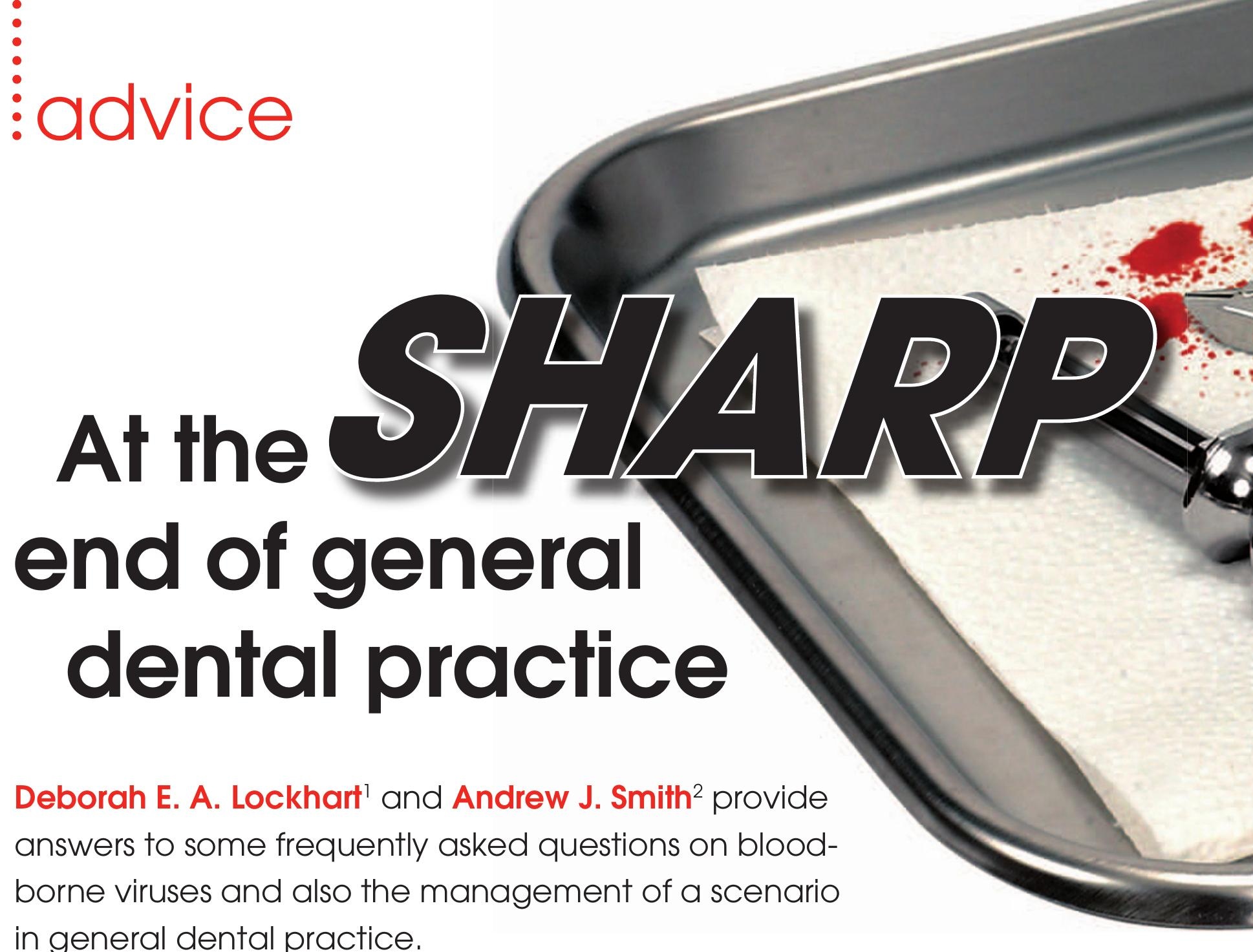

in general dental practice.

${ }^{1}$ Specialist Registrar/Hon Clinical Teacher Microbiology, ${ }^{2}$ Senior Lecturer/Hon Consultant

Microbiologist, Infection Research Group, Level 9, Glasgow Dental Hospital and School,

Faculty of Medicine, 378 Sauchiehall Street, Glasgow G2 3JZ

Email:d.lockhart@dental.gla.ac.uk

\section{Accidents happen}

The waiting room is full of anxious patients and in the surgery a dentist is in the midst of an extraction. Meanwhile, whilst disposing of a local anaesthetic needle you accidentally stab your finger with the used needle. As a member of the dental team you can probably relate to this scenario. But are you confident of the most appropriate means of managing the injury? Or what infectious agents may pose a threat? This article provides answers to some frequently asked questions relating to bloodborne viruses (BBVs) and the management of this injury in general dental practice.

\section{Frequently asked questions}

What are BBVs and why are they

of concern?

BBVs are a group of viruses sharing a common mode of transmission between hosts via blood-to-blood contact. Exposure to BBVs such as hepatitis B (HBV), hepatitis C
(HCV) and human immunodeficiency virus (HIV) pose an occupational risk for all members of the dental team engaged in clinical practice.

Prevention and treatment of BBVS

There is an effective vaccine for HBV and immunisation is compulsory for all staff in direct contact with blood. ${ }^{1}$ Post exposure prophylaxis (PEP) as vaccine +/- immunoglobulin (HBIG) is available for poor responders or those who aren't vaccinated. ${ }^{2}$
No vaccine or PEP exists for HCV but there are effective treatments. Appropriate followup and monitoring is essential to initiate treatment should infection occur following a sharps injury.

HIV has no vaccine or cure, although the introduction of highly active anti-retroviral therapy (HAART) produces long term suppression of the virus. PEP is available and should be commenced ideally within one hour. The decision to prescribe PEP is complex and requires expert input. Percutaneous

\begin{tabular}{l} 
Table 1 Injuries associated with increased risk of HIV transmission ${ }^{5}$ \\
Types of injuries with high risk of transmitting HIV \\
\hline Deep injury \\
Penetrating injury by device visibly contaminated with blood \\
Injury involving placement of needle directly in source patient's artery or vein \\
Exposure to a source patient with end-stage HIV-related ilness
\end{tabular}



Fig. 1 Immediate management of a sharps injury ${ }^{12,13}$

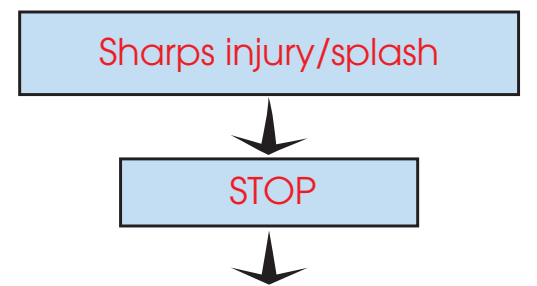

Apply first aid

Encourage bleeding of accidental puncture wounds by gently squeezing.

Do not suck the area.

Wash the affected area with soap and warm water.

Do not scrub the area.

Treat mucosal surfaces such as mouth or conjunctiva by rinsing with warm water or saline.

Do not swallow water used for rinsing the mouth.

Cover injury with waterproof plaster (if applicable).
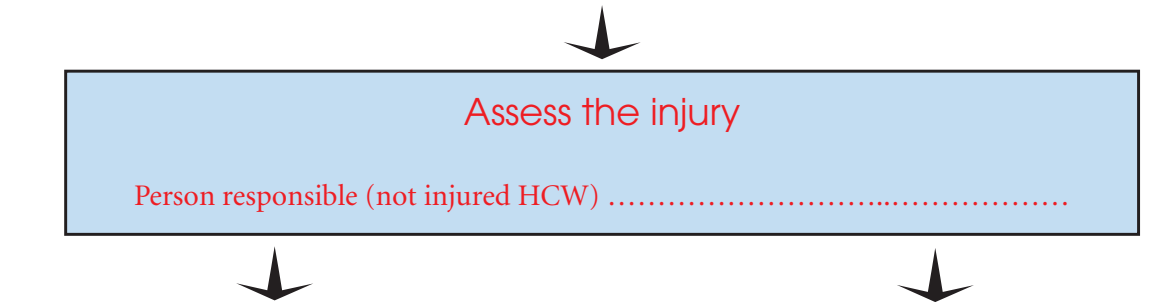

Significant injury

- Percutaneous injury (break of intact skin) from a needle or sharp object in contact with blood or other high risk body fluid*.

- Splashing of blood/high risk body fluid $^{\star}$ onto broken skin or mucous membranes (eyes, nose and mouth).

*Saliva, associated with dentistry, is a high risk body fluid as it is assumed to be contaminated with blood.

\section{$\perp$}

\section{Report and seek immediate expert advice}

Report injury to

Obtain expert advice from one of the following telephone sources: (Each practice to complete)

Occupational Health:. A\&E: .

Doctor:

Have the following information ready:

- Description of injury:.

- HBV immune status: Protected $\square$

Incomplete protection $\square$

Unprotected $\square$

Guidance will be given on conducting the source patient risk assessment (carried out by a senior member of the dental team) i.e. has patient ever had HBV, HCV or HIV or known to be at risk e.g. injected drugs.

Establish arrangements for taking baseline blood samples (HCW and patient).

The need for appropriate PEP and follow-up will be determined.

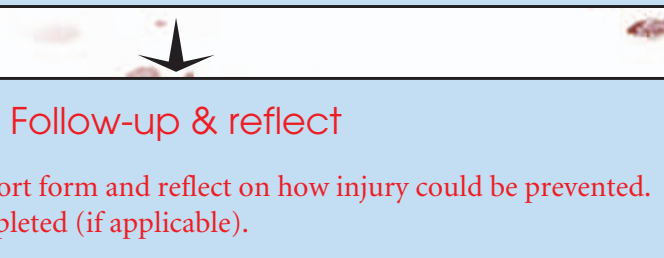

- Complete incident report form and reflect on how injury could be prevented.

- Ensure follow-up completed (if applicable). 


\section{advice}

3. Where to go for treatment and follow-up

4. How to report the incident.

This information should be readily accessible and displayed as a poster in each clinical area. Where possible follow your local primary care trust policies or alternatively adopt the models supplied by national bodies ${ }^{10}$ or the one provided (Fig. 1). There should be a designated person responsible for co-ordinating the management of sharps injuries and maintaining a record of the HBV immune status of staff.

\section{Management of a scenario}

\section{Immediate management}

The nurse should stop the procedure, remain calm and institute immediate first aid measures (Fig. 1). The injury should be assessed by a second person, for example, the dentist. Having classified the injury as 'significant' immediate expert advice should be sought. Meanwhile, the HBV immune status records indicated the nurse was a poor responder to the vaccine (unprotected).

In conjunction with expert advice, a source patient risk assessment performed by the dentist revealed a former intravenous drug user positive for HCV and HIV. The patient denied ever having HBV but was willing to provide a blood sample for testing.

\section{Treatment and follow-up}

Both the nurse and patient attended $A \& E$ for baseline blood sampling. As the patient's HBV status was unknown and the nurse unprotected, she was given HBIG and a booster dose of vaccine.

Careful consideration was given to HIV PEP but the injury in this case was not deemed high risk since the local anaesthetic needle was not placed in the source patient's artery or vein (Table 1). Despite being offered PEP the nurse declined on the grounds of the low risk of transmission and drug side effects. Regular follow-ups and blood tests were negative for HCV and HIV.

\section{Prevention}

Having completed an obligatory incident report ${ }^{11}$ reflection revealed the incident and associated anxiety could have been avoided by adopting some of the preventative strategies outlined.

\section{Conclusion}

Remember that the prevention of sharps injuries is essential, but as accidents may take place, preparation is vital. It is an extremely beneficial exercise for the dental team to undertake role rehearsals and act out different scenarios involving sharps injuries; this may even involve contacting your local A\&E department to confirm that they are your point of reference in the event of an injury. This will mean that all staff are rehearsed in dealing with an incident if it occurs.

1. Expert Advisory Group on AIDS and the Advisory Group on Hepatitis. Guidance for clinical health care workers: protection against infections with blood-borne viruses. London: Department of Health, 1998.

2. PHLS Hepatitis Subcommittee. Exposure to hepatitis B virus: guidance on postexposure prophylaxis. CDR Review 1992; 2: R97-R101.

3. Epidemiologic notes and reports: outbreak of hepatitis B associated with an oral surgeon - New Hampshire. MMWR 1987; 36: 132-133.

4. Health Protection Agency. Eye of the needle. United Kingdom surveillance of significant occupational exposures to bloodborne viruses in healthcare workers. London: Health Protection Agency, 2006.

5. Department of Health. HIV post-exposure prophylaxis: guidance from the UK chief medical officers' expert advisory group on AIDS. London: Department of Health, 2004.

6. Scottish Executive Health Department. Needlestick injuries: sharpen your awareness. Report of the short life working group in NHS Scotland. Edinburgh: SEHD, 2001.

7. Cleveland J L, Barker L K, Cuny E J, Panlilio A L, National Surveillance System for Health Care Workers Group. Preventing percutaneous injuries among dental health care personnel. J Am Dent Assoc 2007; 138: 169-178.

8. Stewardson D A, McHugh S, Palenik C J, Burke F J. Occupational exposures occurring among dental assistants in a UK dental school. Prim Dent Care 2003; 10: 23-26.

9. Zakrzewska J M, Greenwood I, Jackson J. Introducing safety syringes into a UK dental school - a controlled study. Br Dent J 2001; 27: 88-92.

10. Health Protection Scotland Infection Control Team. Standard infection control precautions. Occupational exposure management, including sharps injuries. Glasgow: HPS, 2007. http:// www.hps.scot.nhs.uk/Search/guidedetail. aspx?id=31223

11. Health and Safety Executive. The reporting of injuries, diseases and dangerous occurrences regulations 1995: guidance for employers in the healthcare sector. Suffolk: HSE, 1998.

12. Smith A J, Cameron S O, Bagg J, Kennedy D. Management of needlestick injuries in general dental practice. Br Dent J 2001; 190: 645-650.

13. Public Health Protection Unit. Management of occupational and nonoccupational exposures to blood-borne viruses. NHS Greater Glasgow \& Clyde, 2007. http://www.nhsggc.org.uk/content/ default.asp?page $=$ s257_4

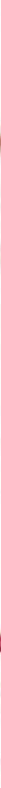

